\title{
Assessment of training effects on autonomic modulation of the cardiovascular system in mature rats using power spectral analysis of heart rate variability
}

\author{
Takashi Kumae
}

Received: 29 September 2011/ Accepted: 15 February 2012/Published online: 11 March 2012

(c) The Japanese Society for Hygiene 2012

\begin{abstract}
Objective To clarify the effects of forced or voluntary exercise on autonomic modulation of the cardiovascular system, we monitored changes in autonomic nervous activity in a mature rat by spectral analysis of the heart rate (HR) during a 10-week training period.

Methods Male Wistar rats implanted with a radio-telemetry system were divided into three groups at 18 weeks of age: (1) Control group $(n=8)$; (2) Voluntary group $(n=6)$, which were housed separately in a cage with a running wheel; (3) Forced group $(n=6)$, which were exercised on a treadmill $(35 \mathrm{~m} / \mathrm{min}, 15 \mathrm{~min} /$ day, 5 days/ week). The electrocardiogram was analyzed by the maximum entropy method into two main oscillations, lowfrequency (LF) and high-frequency (HF) oscillations, respectively. LF and HF are considered to be markers of both sympathetic and parasympathetic modulations and parasympathetic modulation, respectively.

Results Average running distances of the Voluntary group were more than twofold higher than those of the Forced group. HR levels in the Forced group were lower than those in the Control group. LF and HF levels in the Control and the Forced groups were almost the same during the experiment, and those in the Voluntary group showed a tendency to decrease.

Conclusion The results in the Voluntary and the Forced groups suggest that cardiovascular adjustments are not simply caused by the quantity of exercise. In the Voluntary group, both sympathetic and parasympathetic activity may
\end{abstract}

\section{T. Kumae $(\square)$}

Department of Health Promotion and Exercise,

National Institute of Health and Nutrition,

1-23-1 Toyama, Shinjuku-ku, Tokyo 162-8636, Japan

e-mail: kumae@nih.go.jp decrease with a predominance of sympathetic activity. Conversely, in the Forced group, the baroreflex may be hyper-activated by the undesired treadmill running and handling stress.

Keywords Autonomic modulation - Exercise · Heart rate $\cdot$ Rat $\cdot$ Spectral analysis

\section{Introduction}

The overall aim of the research presented in this paper is to prevent chronic fatigue during physical training, especially in sedentary adults starting on an exercise routine for the purpose of personal health promotion. Overtraining due to exercise stress is considered to be one type of chronic fatigue syndrome, and the amount of research into this condition is increasing [1, 2]. Israel [3] differentiates between parasympathetic and sympathetic overtraining, characterized by inhibition or excitation, respectively. Sympathetic overtraining seems to affect primarily speed and power athletes, while parasympathetic overtraining seems to affect mostly endurance athletes [4]. Lehmann et al. [5], in agreement with other investigators [6], reported that changes in blood chemistry are probably not obligatory in parasympathetic overtraining syndromes.

The electrocardiogram (ECG) is much less noninvasive than blood sampling, and modern electronic and computer technology enables the rapid analysis of heart rate variability (HRV) in a frequency domain (spectral analysis) that allows the autonomic modulation of the cardiovascular system to be evaluated [7]. This noninvasive technique has gained considerable interest of researchers and clinicians in the field of both cardiology [8] and neurology [9]. It has since been postulated that the two main oscillations 
observed in the power spectrum, one at a low frequency and the other at a high frequency, may, respectively, be markers of the sympathetic and parasympathetic modulation of the cardiovascular system in rats. Low-frequency power (LF) is considered to be a marker of sympathetic modulation by some investigators $[10,11]$ and by others as a marker of both sympathetic and parasympathetic modulation [7, 12]. High-frequency power (HF) has been accepted to be a marker of parasympathetic modulation [13] and the LF to HF ratio (LF/HF) is often considered as an index of the cardiac sympathovagal balance $[14,15]$.

To estimate parasympathetic overtraining, researchers have studied the power spectrum of heart rate (HR) in freely behaving conscious rats [16]. The study reported here was designed to characterize cardiac autonomic neuropathy in rats subjected to forced or voluntary exercise through the analysis of HRV, based on the hypothesis that the quantitative information provided by this analysis reflects the interaction between sympathetic and parasympathetic regulatory activity [17]. For this purpose, the ECG was recorded from conscious and unrestrained sedentary rats which were not subjected to handling stress through the use of a radio-telemetry system [18]. The ECG recorded by the telemetry system is able to provide more reliable data for the assessment of autonomic nervous activity than data obtained by a tethering system [17]. To clarify the effects of forced or voluntary exercise on the autonomic modulation of the cardiovascular system, we examined changes in autonomic nervous activity and balance during the training period using a mature rat as a model of a sedentary adult $[19,20]$.

\section{Methods}

Experimental animals and protocol

The research protocol was approved by the institutional committee for animal research. Male Wistar JcI strain [specific pathogen free (SPF), 9 weeks old] rats were obtained from Nippon Clea Breeding Laboratories (Tokyo, Japan). The rats were maintained in the SPF condition using an isolated room in a barrier area, as previously reported [16], and kept on a 12/12-h light/dark cycle (the dark cycle was from 6:00 a.m. to 6:00 p.m.). Each rat was implanted with a two-lead ECG radio frequency transmitter (model CA-F40; weight $7.9 \mathrm{~g}$, volume $4.2 \mathrm{~cm}^{3}$; Data Sciences International, Saint Paul, MN) at 15 weeks of age. After a recovery period of 3 weeks (age 18 weeks), the rats were divided into the following three groups: (1) control group (Control, $n=8$ ), housed under the usual breeding condition for rats kept under sedentary conditions; (2) voluntary training group (Voluntary, $n=6$ ), housed separately in a cage with a running wheel (Microtec NitiOn, Chiba, Japan); (3) forced training group (Forced, $n=6$ ); housed as the Control group, but forced to exercise on a treadmill (Suzuya Co, Tokyo, Japan). The Voluntary group had 24-h access to the running wheel (circumference $1 \mathrm{~m}$, width $10 \mathrm{~cm}$ ). The daily running distance of the Voluntary group was measured as rotation counts of the running wheel. To monitor rotation counts without disturbing the voluntary running, the rotation counts were converted to electric signals and transmitted by a cable to another room. To maintain a noiseless environment in the isolated breeding room, the treadmill exercise $(35 \mathrm{~m} / \mathrm{min}$, $15 \mathrm{~min} /$ day, 5 days/week) was performed also in another room at almost the same time on each test day in the dark cycle (around 1:00 p.m.). The duration of the experimental period was 10 weeks. The rats were given rodent feed (F-2; Funabashi Farms, Chiba, Japan) and water ad libitum. The feed weight was measured to calculate feed consumption, and body weights were measured each week.

\section{ECG recording}

The ECG was recorded during the dark cycle in conscious and unrestrained rats at once-weekly intervals at almost the same time (hour of the day) on each occasion. The ECG recording for the Forced group was carried out before the exercise (treadmill running). Care was taken to keep the room noiseless during the ECG recording, and the original ECG signal was received by one set of receiving poles (RLA3000; Data Sciences International) located on both sides of each rat cage. The ECG signal was amplified using a receiver multiplexer (RMX10; Data Sciences International), transmitted to another room by a cable to maintain the noiseless state of the isolated breeding room, further amplified, and then subjected to cut-off noise by a recticorder (RJG-4122, Nihon Koden, Tokyo, Japan) and an electroencephalograph (EEG) (EEG1A94; San-ei Co, Tokyo, Japan). To collect the ECG data for a continuous 15-min interval for each rat without physical movements and/or positional changes, the ECG was monitored with the EEG during the recording. The amplified ECG analogue signals were recorded into a magnetic tape (SIT80; Sony Magnescale, Tokyo, Japan) by an instrumentation tape recorder (A-49; Sony Magnescale, Tokyo, Japan) [16].

\section{Power spectral analysis of HRV}

$R$ wave-to- $R$ wave (RR) intervals in the ECG analogue signals recorded with the tape recorder were analyzed at high resolution [21] by the maximum entropy method (MEM) [22] using special software (Tarawa Mouse \& Rat; Suwa Trust, Tokyo, Japan) in consecutive periods of $10 \mathrm{~s}$ for $15 \mathrm{~min}$ [16]. An accurate estimation of the power 
spectral analysis is possible even with a small number of samples, producing a signal which is supposed to maintain stationary [23]. In this study, 1 min of the lowest HR region of the ECG recorded without physical movements was selected as the stationary part for the power spectral analysis. HRV is represented as an entropy (ETY) in the frequency domain. ETY is calculated by continuous eight pulses of $\mathrm{R}$ waves and expressed as the percentage ratio set at constant RR (0\%); most random variation of RR is $100 \%$. Most researchers agree that there are two major components, i.e., LF and HF, in the power spectra; however, there is as yet no constant definition of the frequency of LF and $\mathrm{HF}$ in rats. In this study, rat LF and HF components were defined as $0.20-0.80$ and $0.8-3.5 \mathrm{~Hz}$, respectively [16]. The HRV components, LF and HF, were log-transformed to normalize their distribution for the statistical tests [24], and the $\mathrm{LF} / \mathrm{HF}$ ratio was calculated as an index of sympathetic and parasympathetic activity balance [14-16].

\section{Statistics}

Two-factor analysis of variance (ANOVA) was employed to establish the effect of the experimental manipulation (factor group), the experimental period (factor week), as well as the interaction effects between factors, on the measured items using StatView software (SAS Institute, Cary, NC). The statistical significance of the differences among the data obtained on each weekly examination was determined by one-factor ANOVA. The statistical significant changes in the data obtained in each group during the experiment were determined by repeated-measures ANOVA. Where a main effect was observed, post hoc test using Dunnett's $t$ was performed for multiple comparisons. Correlation analysis was carried out to examine the relations among indices of the power spectral analysis of HRV in each group during the experiment.

\section{Results}

Changes in body weight, feed consumption, and running distance

Increases in body weight were suppressed by the implantation surgery at age 15 weeks; however, body weight did increase after the 3 -week recovery period. As shown in Fig. 1, 3 weeks following the beginning of the experiment, the mean body weight in the Voluntary group was slightly lower than that in the Control group, and that in the Forced group was lower than that in the Voluntary group. Twofactor ANOVA revealed that this difference in body weight among the groups at 3 weeks was not significant, although the difference between body weight during the experiment

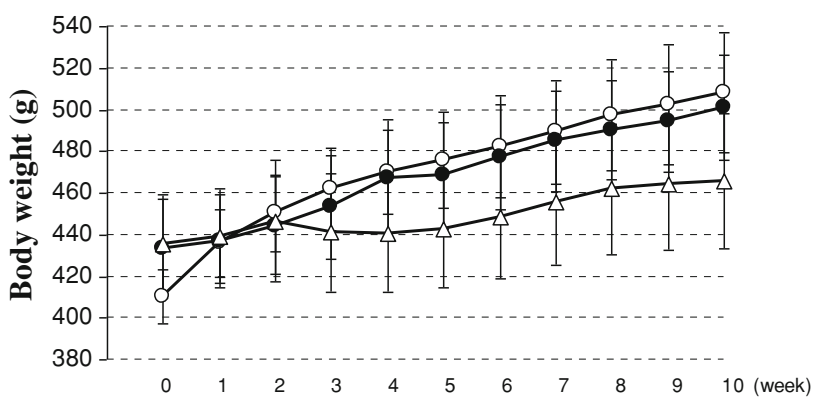

Fig. 1 Changes in body weight during the experiment. Vertical axis Body weight (g). Symbols Mean values of the experimental groups: open circles Control group $(n=8)$, filled circles Voluntary group $(n=6)$, open triangles Forced group $(n=6)$. Vertical bars Standard error (SE). See section Experimental animals and protocol for description of animal groups

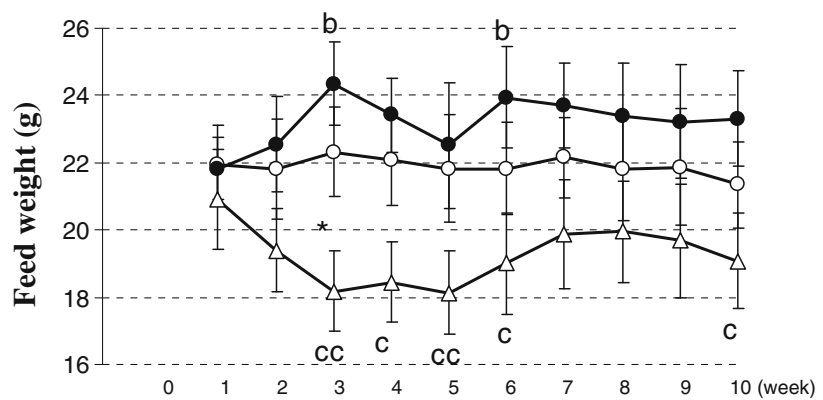

Fig. 2 Changes in feed consumption during the experiment. Vertical axis Weight of consumed feed (g). Asterisk Statistical difference $(p<0.05)$ between the Control group and other groups at the same time-point, as revealed by analysis of variance (ANOVA). $b$ denotes statistical difference $(p<0.05)$ in feed consumption in the Voluntary group between week 1 and corresponding weeks as revealed by repeated-measures ANOVA, $c, c c$ statistical differences $(p<0.05$ and $p<0.01$, respectively) in feed consumption in the Forced group between week 1 and corresponding weeks as revealed by repeatedmeasures ANOVA. Symbols and vertical bars are the same as for Fig. 1

was significant $(p<0.05)$. There was no significant difference in mean body weight among the groups, and no significant change during the experiment within each group (Fig. 1).

Changes in the feed consumption are shown in Fig. 2. Compared to the mean level of feed consumption in the Control group at experimental week 2, mean feed consumption was higher in the Voluntary group and lower in the Forced group. Two-factor ANOVA revealed a significant difference in the feed consumption among the groups $(p<0.001)$ without any significant difference during the experimental period. The Forced group consumed significantly $(p<0.05)$ small amounts of feed than the Control group at Week 3 when the mean body weight in the Forced group showed a tendency to decrease (Fig. 1). The Voluntary group showed a significant increase in mean feed consumption between week 1 and week 3 and between 


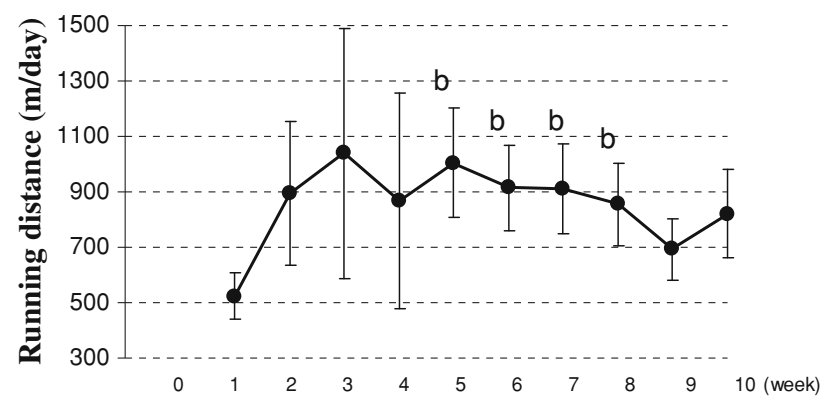

Fig. 3 Changes in mean running distance in the Voluntary group. Vertical axis Running distance (m/day). $b$ denotes statistically significant difference $(p<0.05)$ in mean running distance in the Voluntary group between in week 1 and weeks denoted by $b$, as revealed by repeated-measures ANOVA. Symbols and vertical bars are the same as in Fig. 1

week 1 and week 6 ( $p<0.05$ for both). In contrast, the Forced group showed decreased feed consumption between week 1 and weeks 3, 4, 5, 6, and 10 (Fig. 2).

The running distance of the Forced group was kept as $525 \mathrm{~m} /$ day $(35 \mathrm{~m} / \mathrm{min}, 15 \mathrm{~min} /$ day, 5 days/week) during the experiment. As shown in Fig. 3, the Voluntary group run almost the same distance as the Forced group during week 1 ( $524 \mathrm{~m} /$ day), but significant increases in the mean running distance were observed between week 1 and week 5 up to week 8 ( $p<0.05$ for all). The Voluntary group run an average around $800 \mathrm{~m} /$ day/week during the experiment.

Changes in the mean levels of HR and ETY

Changes in the mean HR level are shown in Fig. 4. Compared to the Control group, the mean HR level was higher in the Voluntary group and lower in the Forced group. Twofactor ANOVA revealed a significant difference in the HR level among the groups $(p<0.001)$, with a significant difference during the experimental period $(p<0.01)$. A significant difference in HR level was observed between the Control and Forced groups at week $1(p<0.05)$ and between the Control and the Voluntary groups at weeks 7 and 9 ( $p<0.05$ and $p<0.001$, respectively) (Fig. 4). The mean HR level in the Control group significantly decreased between week 0 and week $5(p<0.05)$. Compared to week 0 , the Forced group showed decreased levels of HR during weeks 5, 9, and 10 ( $p<0.05$ for all) (Fig. 4).

Contrary to the changes in the HR, there was no constant trend with respect to changes in the ETY level (Fig. 5). In the Voluntary group, mean levels of ETY showed a tendency to decrease after week 6; however, no significant difference among the groups and during the experimental period was observed by two-factor ANOVA. Repeatedmeasures ANOVA revealed a significant difference in the mean level of ETY between week 0 and week $6(p<0.05)$ in the Voluntary group.

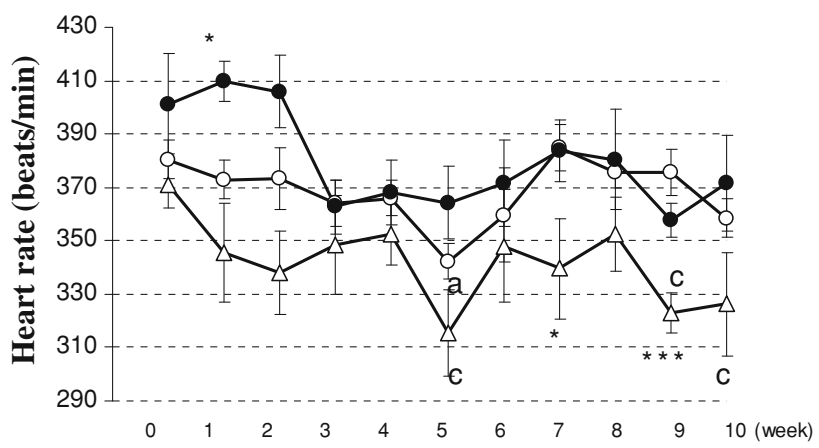

Fig. 4 Changes in the mean heart rate (HR). Vertical axis Heart rate (beats/min). Single and triple asterisks denote statistical difference ( $p<0.05$ and $p<0.001$, respectively) in mean HR between the Control group and corresponding groups at that time-point as revealed by ANOVA. $a, c$ denote statistical difference $(p<0.05$ for all $)$ in mean HR between week 0 and the corresponding weeks, as revealed by repeated measures in the Control and the Forced groups, respectively. Symbols and vertical bars are the same as in Fig. 1

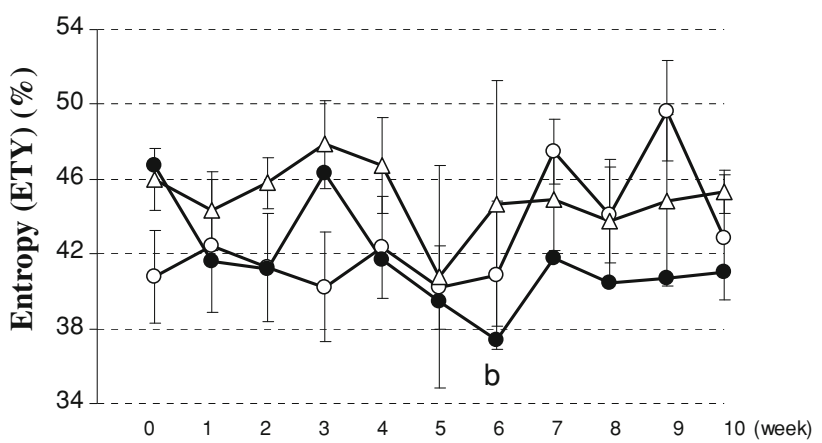

Fig. 5 Changes in the mean level of the entropy (ETY) during the experiment. Vertical axis Entropy (\%). $b$ denotes statistical difference $(p<0.05)$ in mean ETY in the Voluntary group between week 0 and week 6 as revealed by repeated-measures ANOVA. Symbols and vertical bars are the same as in Fig. 1

\section{Changes in indices of power spectral analysis of HRV}

The LF levels in the Control and Forced groups remained almost at the same levels during the experimental period and those in the Voluntary group showed a tendency to decrease after week 4 (Fig. 6). No significant difference among the groups and during the experimental period was revealed by two-factor ANOVA. A significant difference in the mean LF was observed between week 0 and week 10 $(p<0.05)$ in the Voluntary group by repeated-measures ANOVA.

Changes in mean levels of HF are shown Fig. 7. The changes of HF were similar to those of LF but mean levels in the Voluntary group showed apparent decreased tendency than the changes of LF (Fig. 6). Two-factor ANOVA revealed significant difference in mean levels of $\mathrm{HF}$ among the groups $(p<0.01)$ without significant difference during the experimental period. Mean level of HF 


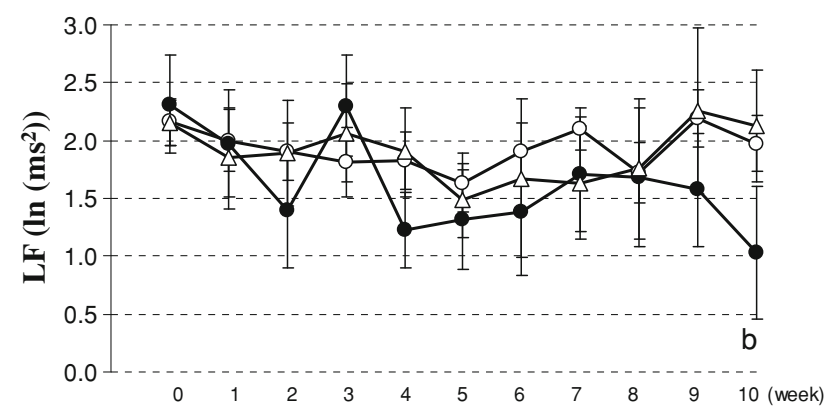

Fig. 6 Change in mean low-frequency $(L F)$ oscillations during the experimental period. Vertical axis Natural logarithms of spectral powers in the LF region $\left(\mathrm{m} \mathrm{s}^{2}\right)$. $b$ denotes statistical difference $(p<0.05)$ between mean LF in the Voluntary group at week 0 and week 10 as revealed by repeated-measures ANOVA. Symbols and vertical bars are the same as in Fig. 1

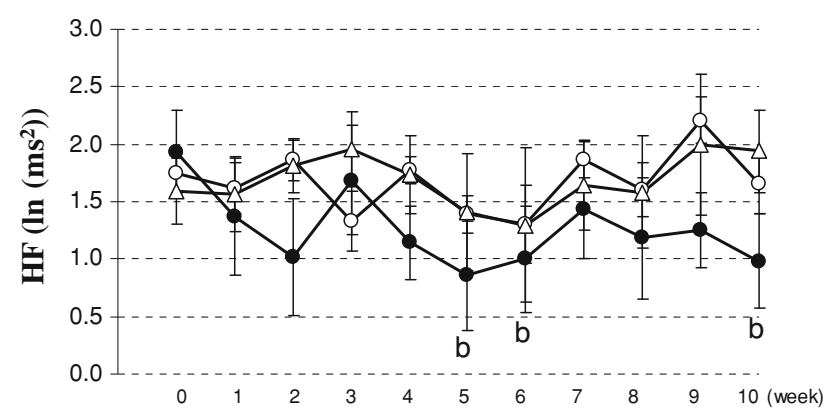

Fig. 7 Change in mean high-frequency (HF) oscillations during the experiment. Vertical axis Natural logarithms of spectral powers in the $\mathrm{HF}$ region $\left(\mathrm{m} \mathrm{s}^{2}\right) . b$ denotes statistical difference $(p<0.05)$ between mean HF in the Voluntary group at week 0 and corresponding weeks as revealed by repeated measures. Symbols and vertical bars are the same as in Fig. 1

at week 0 was significantly deceased at week 5 , week 6 , and week $10(p<0.05$ for all $)$ in the Voluntary group revealed by the repeated measures.

As shown in Fig. 8, the mean level of LF/HF seemed to fluctuate differently in each group. However, two-factor ANOVA revealed no significant difference in the mean LF/ HF among the groups, although there was a significant difference during the experimental period $(p<0.05)$. There was no significant difference in the mean $\mathrm{LF} / \mathrm{HF}$ among the groups and no significant change during the experiment in each group (Fig. 8).

Correlations among HR, ETY, and indices of power spectral analysis of HRV

The correlation coefficients and statistical significances are summarized in Table 1. There was no significant correlation between HR and ETY, between HR and LF/HF, and between ETY and LF/HF in all groups. To the contrary, significant positive correlations were observed between

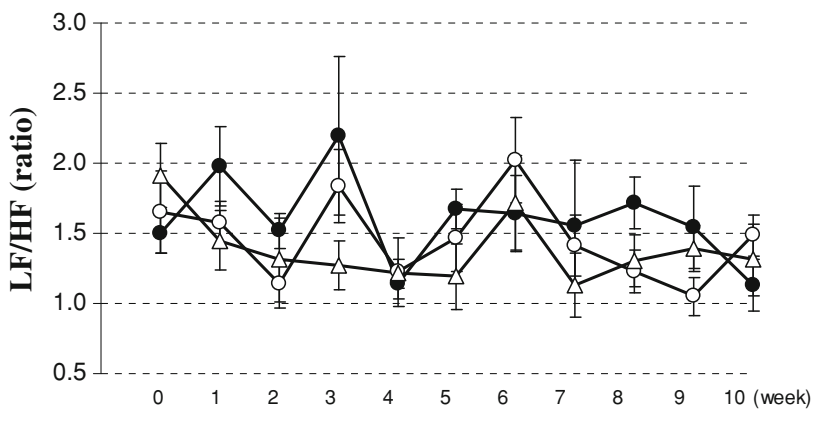

Fig. 8 Changes of mean levels of the LF/HF during the experiment. Vertical axis is the LF/HF (ratio). Symbols and vertical bars are same as Fig. 1

ETY and HF and between LF and HF in all groups. In the Control group, HR significantly positively correlated to LF and HF ( $p<0.05$ for both), and a significant negative correlation was observed between HF and LF/HF. Significant positive correlations were observed between ETY and LF in the Voluntary and Forced groups $(p<0.01$ and $p<0.05$, respectively). In the Voluntary group, LF significantly positively correlated to LF/HF $(p<0.05)$.

\section{Discussion}

In this study, running distances were different between the Forced and Voluntary groups due to the intrinsic difficulty in the experimental set-up of regulating the voluntary running [25, 26]. As shown in Fig. 3, although the running distance of the Voluntary group was almost same that of the Forced group at the week 1 time-point, it showed a tendency to increase from week 2 onwards in the Voluntary group. It was expected that the running distance would settle down to the level of week 1 with practice; consequently, the treadmill running time in the Forced group was not able to increase as rapidly in accordance with the running distance of the Voluntary group for the prevention of stragglers in the Forced group [20]. The mean running distance of the Voluntary group was almost twofold that of the Forced group on any one day per week. The absence of a tendency for the Voluntary group to increase body weight (Fig. 1) suggests that the increase in energy expenditure through the voluntary running is compensated by increased feed consumption (Fig. 2). It has been reported that voluntary wheel running does not modify body weight gain and increased total caloric intake [27]. The tendency for body weights to decrease in the Forced group may be caused by decreased feed consumption. These results are in accordance with those of our previous study [20]. It has also been reported that rats exercising on a treadmill eat less food than resting animals [28] and that body weight losses that could be accounted for entirely by a reduction in feed intake [29]. 
Table 1 Correlations among heart rate, entropy, and indices of the power spectral analysis of heart rate variability
$* p<0.05, * * p<0.01$,

$* * * p<0.001$

ETY Entropy, HR heart rate,

$L F$ low-frequency oscillation, $H F$ high-frequency oscillation

LF and HF were log-

transformed to normalize their distribution for statistical tests

\begin{tabular}{llllr}
\hline Variables/experimental group & ETY & LF & HF & LF/HF \\
\hline HR & & & & \\
Control & 0.534 & $0.675^{*}$ & $0.628^{*}$ & -0.294 \\
Voluntary & 0.223 & 0.333 & 0.285 & 0.098 \\
Forced & 0.540 & 0.173 & -0.200 & 0.571 \\
ETY & & & & \\
Control & - & 0.563 & $0.776^{* *}$ & -0.574 \\
Voluntary & - & $0.767^{* *}$ & $0.882^{* * *}$ & 0.252 \\
Forced & - & $0.645^{*}$ & $0.588^{*}$ & 0.144 \\
LF & - & & & -0.075 \\
Control & - & - & $0.670^{*}$ & $0.697^{*}$ \\
Voluntary & - & - & $0.911^{* * *}$ & 0.314 \\
Forced & - & - & $0.772^{* *}$ & \\
HF & - & - & - & $-0.784^{* *}$ \\
Control & - & - & - & 0.367 \\
Voluntary & - & & -0.335 \\
Forced & & & & \\
\hline
\end{tabular}

It has been reported that when working with conscious unrestrained animals, the limiting factor in the selection of the data to be analyzed was that the animal be quiet [23]. In previous studies, stationary sets of data of the appropriate time span have often been selected for analysis without any clear definition [30, 31]. In this study, for a fair and impartial selection of stationary datasets, $1 \mathrm{~min}$ of the lowest HR region was analyzed by high-resolution MEM in consecutive periods of $10 \mathrm{~s}[21,22]$. It is well known that endurance training induces bradycardia and a reduction in the resting HR. Many investigators have examined the sympathetic and parasympathetic effects on the heart to explain the bradycardia resulting from training. Baroreceptor signaling has been reported to play a decisive role in driving treadmill running-induced cardiovascular adjustments using rats with a chronic absence of baroreceptor inputted by sino-aortic denervation [32]. However, according to the results of this study, average running distances of the Voluntary group were more than twofold higher than those of the Forced group without any observed reduction in the HR (Fig. 4). These results suggest that cardiovascular adjustments are not simply caused by exercise quantity alone. Body weight, HR, and systolic and diastolic blood pressure have all been found to decrease in response to dietary restriction in male Sprague-Dawley rats [33]. The beat-to-beat interval of HR has also been reported to be significantly reduced by elevated plasma nonesterified fatty acids levels [34]. Both treadmill and voluntary wheel running exercise has been found to decrease the plasma concentrations of free fatty acids in diabetic rats [35] and Wistar/ST rats [36]. Consequently, it is possible that the reduced feed consumption and decreased level of plasma free fatty acids due to the exercise regimen contributed to the observed reduction in HR in the Forced group.

The absence of a significant correlation between HR and ETY in all groups (Table 1) suggests that HR is independent of ETY representing HRV. Increased levels of ETY mean that the HRV varied more randomly and give an impression that the power spectra are increased; however, theoretically, ETY is independent of components of the power spectra [22]. In this study, significant positive correlations were observed between ETY and components of the power spectra-with the exception of LF in the Control group (Table 1). The ETY levels showed no constant trend in changes and no significant difference among the groups (Fig. 5). These results suggest that the observed positive correlations are caused by time-course effects of exaggerated cardiovascular responses to exercise, both the voluntary and forced exercise, on ETY and components of the power spectra.

The LF components of HRV in rats are reduced by cardiac parasympathetic or sympathetic blockade [14, 17], and whereas sympathetic blockade has no significant effect on the HF components [17], cardiac parasympathetic blockade abolishes the HF components [37]. According to these studies, LF in rats can therefore be considered to be a marker of both sympathetic and parasympathetic modulations and $\mathrm{HF}$ as a marker of parasympathetic modulation. In this study, no significant difference among the groups and during the experimental period in mean LF was revealed by two-factor ANOVA. To the contrary, mean levels of HF in the Voluntary group showed a tendency to decrease after week 4 (Fig. 7), when a significant 
difference among the groups was observed. According to these results, cardiac parasympathetic modulation is suppressed in the Voluntary group. However, there is no significant difference among the groups in mean levels of LF/HF (Fig. 8) which is the convenient index of cardiac sympathovagal balance [14-16].

In this study, ECG was recorded during the dark cycle when rats were expected to be awake and active. It has been reported that, in rats, LF and HF in the dark cycle are lower than those in the light cycle and that LF/HF in the dark cycle is significantly higher than that in the light cycle [38]. The lower HF levels observed in the Voluntary group suggest that the Voluntary group is awake longer and more active than other groups in the dark cycle. There is another interpretation for the lower HF levels. It has been shown that HF is dependent on respiratory rate [37]. The Voluntary group was housed separately in a cage with a running wheel. Respiratory rate was not measured in this study; however, compared to animals housed in pairs, isolated rats have been shown to have a similar HR and to breathe faster [39]. The relative role of parasympathetic, sympathetic, and ventilatory influences on HRV is controversial [40]. In rats, mild stress-elicited increases in HR have been associated with spectral modifications reflecting sympathetic hyperactivity, i.e., an increase in LF and a decrease in HF [41]. In one study, a significant increase in LF was observed by treadmill exercise in a dog [42], and in another study the LF/HF ratio was augmented whenever the sympathovagal balance was shifted toward sympathetic predominance with mild physical exercise or mental stress [11]. In this study, ECG was measured before the treadmill exercise, which was assumed to the reasons for there being no significant difference in the mean $\mathrm{LF}$ and $\mathrm{LF} / \mathrm{HF}$ ratio among the groups.

It also has been suggested that vagal activation is independent of sympathetic activation [43]. LF and HF cannot be interpreted as a reciprocal or 'push-pull' relationship between sympathetic and parasympathetic control [44]. Significant positive correlations were observed between LF and HF in all groups (Table 1), suggesting that the relationship between LF and HF is not a simple reciprocal relationship and that changes of the $\mathrm{LF}$ and $\mathrm{HF}$ levels occur simultaneously in the time-course of the experiment. In the Voluntary group, the HF levels significantly decreased, but the $\mathrm{HR}$ and $\mathrm{LF} / \mathrm{HF}$ remained at almost Control group levels, indicating that both sympathetic and parasympathetic activity decreased, sympathetic activity predominating. To the contrary, bradycardia was observed in the Forced group without any significant difference in the power spectral analysis, indicating that both sympathetic and parasympathetic activity increased, with parasympathetic activity predominating. Although the mean running distance in the Forced group was almost half that in the Voluntary group, the Forced group is thought to be in the overtraining situation due to the forced training and handling stress [18]. The baroreflex in the Forced group may be hyper-activated to adapt to an acute increase of blood pressure caused by the undesired treadmill running. Inhibitory vagal effects on food intake have been demonstrated using vagotomized rats [45]; therefore, decreased levels of feed intake in the Forced group may be caused by increased levels of parasympathetic activity.

More detailed studies with an increased number of animals are needed to clarify the effects of exercise on the autonomic modulation of the cardiovascular system. It is difficult to extrapolate the present findings in rats to humans because of the marked differences in the physiological characteristics between rodents and humans. However, the results obtained in this study suggest that the exercise for health promotion should be performed voluntarily to prevent chronic fatigue.

Acknowledgments This work was supported in part by research grants from the Ministry of Education, Science and Culture of Japan (no. 14380022). The author wishes to thank the staff of the Department of Industrial Health, National Institute of Public Health for their contributions.

Conflict of interest None.

\section{References}

1. Sharp NCC, Koutedakis Y. Sport and the overtraining syndrome: Immunological aspects. Br Med Bull. 1992;48:518-33.

2. Budgett R. The overtraining syndrome. Br Med J. 1994;309: 465-8.

3. Israel S. Zur Problematik des Übertraining aus internistischer und leistungsphysiologischer 0 Sicht. Med Sport. 1976;16:1-12.

4. Hooper SL, MacKinnon LT. Monitoring overtraining in athletes: recommendations. Sports Med. 1995;20:321-7.

5. Lehmann M, Dickhuth HH, Gendrisch G, Lazar W, Thum M, Kaminski R, et al. Training-overtraining. A prospective, experimental study with experienced middle- and long-distance runners. Int J Sports Med. 1991;12:444-52.

6. Kuipers H, Keizer HA. Overtraining in elite athletes: review and directions for the future. Sports Med. 1988;6:79-92.

7. Akselrod S, Gordon D, Ubel FA, Shannon DC, Barger AC, Cohen RJ. Power spectrum analysis of heart rate fluctuation: a quantitative probe of beat-to-beat cardiovascular control. Science. 1981;213:220-2.

8. Malik M, Camm AJ. Heart rate variability and clinical cardiology. Br Heart J. 1994;71:3-6.

9. Donchin J, Constantini S, Szold A, Byrne EA, Porges SW. Cardiac vagal tone predicts outcome in neurosurgical patients. Crit Care Med. 1992;20:942-9.

10. Kamath MV, Fallen EL. Power spectral analysis of heart rate variability: a noninvasive signature of cardiac autonomic function. Crit Rev Biomed Engl. 1993;21:245-311.

11. Montano N, Gnecchi-Ruscone T, Porta A, Lombardi F, Pagani M, Malliani A. Power spectrum analysis of heart rate variability to assess the changes in sympathovagal balance during graded orthostatic tilt. Circulation. 1994;90:1826-31. 
12. Appel ML, Berger RD, Saul JP, Smith JM, Cohen RJ. Beat to beat variability in cardiovascular variables: noise or music ? J Am Coll Cardiol. 1989;14:1139-48.

13. Perlstein I, Stepensky D, Sapoznikov D, Hoffman A. Power spectral analysis of heart rate variability in rats as a quantitative tool in the PK-PD analysis of the parasympatholytic activity of atropine. Pharm Res. 2001;18:1220-5.

14. Rimoldi O, Pierini S, Ferrari A, Cerutti S, Pagani M, Malliani A. Analysis of short-term oscillations of R-R and arterial pressure in conscious dogs. Am J Physiol. 1990;258(4 Pt 2):H967-76.

15. Cerutti C, Barres C, Paulter C. Baroreflex modulation of blood pressure and heart rate variabilities in rats: assessment by spectral analysis. Am J Physiol. 1994;266(5 Pt 2):H1993-2000.

16. Kumae T, Arakawa H. A study for assessment of day-night cycle disturbance effects on the autonomic modulation in freely behaving rats using spectral analysis of heart rate variability (article in Japanese). J Phys Fit Neut Immun. 2003;13:112-20.

17. Kuwahara M, Yayou $\mathrm{K}$, Ishii $\mathrm{K}$, Hashimoto $\mathrm{S}$, Tsubone $\mathrm{H}$, Sugano S. Power spectral analysis of heart rate variability as a new method for assessing autonomic activity in the rat. J Electrocardiol. 1991;27:333-7.

18. Hashimoto M, Harada T, Ishikawa T, Obata M, Shibutani Y. Investigation on diabetic autonomic neuropathy assessed by power spectral analysis of heart rate variability in WBN/Kob rats. J Electrocardiol. 2001;34:243-50.

19. Kiss A, Delattre AM, Pereira SI, Carolino RG, Szawka RE, Anselmo-Franci JA, et al. $17 \beta$-Estradiol replacement in young, adult and middle-aged female ovariectomized rats promotes improvement of spatial reference memory and an antidepressant effect and alters monoamines and BDNF levels in memory- and depression-related brain areas. Behav Brain Res. 2011;227:100-8.

20. Kumae T, Arakawa H. Assessment of training effects on activity levels of alveolar macrophage in matured rats using chemiluminescent technique. Luminescence. 2003;18:61-6.

21. Murasato Y, Hirakawa H, Harada Y, Nakamura T, Hayashida Y. Effects of systemic hypoxia on R-R interval and blood pressure variabilities in conscious rats. Am J Physiol. 1998;275(3 Pt 2): H797-804.

22. Saito K, editor. A recent advance in time series analysis by maximum entropy method; application to medical and biological sciences (article in Japanese). Hokkaido: Hokkaido University Press; 1994.

23. Malik M. Heart rate variability: standards of measurement, physiological interpretation and clinical use. Task Force of the European Society of cardiology and the North American Society of Pacing and Electrophysiology. Circulation 1996;93:1043-65.

24. Gehi A, Lampert R, Veledar E, Lee F, Goldberg J, Jones L, et al. A twin study of metabolic syndrome and autonomic tone. J Cardiovasc Electrophysiol. 2009;20:422-8.

25. Novelli M, Pocai A, Skalicky M, Viidik A, Bergamini E, Masiello P. Effects of life-long exercise on circulating free fatty acids and muscle triglyceride content in ageing rats. Exp Gerontol. 2004;39:1333-40.

26. Narath E, Skalicky M, Viidik A. Voluntary and forced exercise influence the survival and body composition of ageing male rats differently. Exp Gerontol. 2001;36:1699-711.

27. Oudot F, Larue-Achagiotis C, Anton G, Verger P. Modifications in dietary self-selection specifically attributable to voluntary wheel running and exercise training in the rat. Physiol Behav. 1996;59:1123-8.

28. Rivest S, Richard D. Involvement of corticotropin-releasing factor in the anorexia induced by exercise. Brain Res Bull. 1990;25:169-72.
29. Dulloo AG, Miller DS. The effect of parasympathetic drugs on energy expenditure: relevance to the autonomic hypothesis. Can J Physiol Pharmacol. 1986;64:586-91.

30. Daffonchio A, Franzelli C, Di Rienzo M, Castiglioni P, Mancia G, Ferrari AU. Sympathetic, parasympathetic and non-autonomic contributions to cardiovascular spectral powers in unanesthetized spontaneously hypertensive rats. J Hypertens. 1994;13:1636-42.

31. Aubert AE, Ramaekers D, Beckers F, Breem R, Denef C, Van de Werf $F$, et al. The analysis of heart rate variability in unrestrained rats. Validation of method and results. Comput Methods Progr Biomed. 1999;60:197-213.

32. Ceroni A, Chaar LJ, Bombein RL, Michelini LC. Chronic absence of baroreceptor inputs prevents training-induced cardiovascular adjustments in normotensive and spontaneously hypertensive rats. Exp Physiol. 2009;94:630-40.

33. Mager DE, Wan R, Brown M, Cheng A, Wareski P, Abernethy $\mathrm{DR}$, et al. Caloric restriction and intermittent fasting alter spectral measures of heart rate and blood pressure variability in rats. FASEB J. 2006;20:631-7.

34. Shaltout HA, Abdel-Rahman AA. Mechanism of fatty acids induced suppression of cardiovascular reflexes in rats. J Pharmacol Exp Ther. 2005;314:1328-37.

35. Belotto MF, Magdalon J, Rodrigues HG, Vinolo MA, Curi R, Pithon-Curi TC, et al. Moderate exercise improves leucocyte function and decreases inflammation in diabetes. Clin Exp Immunol. 2010;162:237-43.

36. Hagio M, Matsumoto M, Yajima T, Hara H, Ishizuka S. Voluntary wheel running exercise and dietary lactose concomitantly reduce proportion of secondary bile acids in rat feces. J Appl Physiol. 2010;109:663-8.

37. Cerutti C, Gustin MP, Paultre CZ, Lo M, Julien C, Vincent M, et al. Autonomic nervous system and cardiovascular variability in rats: a spectral analysis approach. Am J Physiol. 1991;261(4 Pt 2): H1292-9.

38. Hashimoto M, Kuwahara M, Tsubone H, Sugano S. Diurnal variation of autonomic nervous activity in the rat: investigation by power spectral analysis of heart rate variability. J Electrocardiol. 1999;32:167-71.

39. Blanc J, Baudrie V, Tulen J, Ponchon P, Gaudet E, Elghozi JL. Social isolation affects the pattern of cardiovascular responses to repetitive acoustic startle stimuli. Clin Exp Pharmacol Physiol. 1997;24:40-5.

40. Perlini S, Giangregorio F, Coco M, Radaelli A, Solda PL, Bernardi L, et al. Autonomic and ventilatory components of heart rate and blood pressure variability in freely behaving rats. Am J Physiol. 1995;269(5 Pt 2):H1729-34.

41. Blanc J, Grichois ML, Elghozi JL. Effects of clonidine on blood pressure and heart rate responses to an emotional stress in the rat: a spectral study. Clin Exp Pharmacol Physiol. 1991;18:711-7.

42. Malliani A, Pagani M, Lomardi F, Cerruti S. Cardiovascular neural regulation explored in the frequency domain. Circulation. 1991;84:482-92.

43. Rissanen P, Franssila-Kallunki A, Rissanen A. Cardiac parasympathetic activity is increased by weight loss in healthy obese women. Obes Res. 2001;9:637-43.

44. Bernardi M, Deslauriers R, Docherty J, Galeazzi G, Rossini L, Rossini P. Spectral analysis of intercycle heart fluctuations in the diethyl-ether-anaesthetized or pithed rat treated with 1-hyoscyamine. J Auton Pharmacol. 1997;17:27-34.

45. Yang ZJ, Ratto C, Gleason JR, Bellantone R, Crucitti F, Meguid MM. Influence of anterior subdiaphragmatic vagotomy and TPN on rat feeding behavior. Physiol Behav. 1992;51:919-26. 